\title{
Spatio-temporal anomalous diffusion imaging: results in controlled phantoms and in excised human meningiomas
}

\author{
Silvia Capuani a,b,*, Marco Palombo a,b, Andrea Gabrielli ${ }^{\text {c }}$, Augusto Orlandi ${ }^{\text {d }}$, Bruno Maraviglia e,f, \\ Francesco S. Pastore ${ }^{g}$ \\ a Physics Department, Sapienza University of Rome, P.le Aldo Moro, 500185 Rome, Italy \\ b CNR-IPCF UOS Roma Sapienza, Physics Department, Sapienza University of Rome, P.le Aldo Moro, 500185 Rome, Italy \\ c ISC-CNR via dei Taurini, 1900185 Rome, Italy \\ d Biopathology and Image Diagnostics, "Tor Vergata" University of Rome, Italy \\ e Neuroimaging Laboratory, Santa Lucia Foundation, Via Ardeatina 306, 00179 Rome, Italy \\ ${ }^{\mathrm{f}}$ Enrico Fermi Center, Rome, Italy \\ ${ }^{g}$ Department of Neurosurgery, "Tor Vergata” University of Rome, Italy
}

\section{A R T I C L E I N F O}

\section{Article history:}

Received 18 November 2011

Revised 6 August 2012

Accepted 30 August 2012

Available online $\mathrm{xxxx}$

\section{Keywords:}

Stretched exponential model

Diffusion time variable PGSTE

Anomalous diffusion

Non-Gaussian diffusion

Magnetic susceptibility differences

Disorder degree

Polystyrene micro-beads

Meningiomas

\begin{abstract}
A B S T R A C T
Recently, we measured two anomalous diffusion (AD) parameters: the spatial and the temporal AD indices, called $\gamma$ and $\alpha$, respectively, by using spectroscopic pulse gradient field methods. We showed that $\gamma$ quantifies pseudo-superdiffusion processes, while $\alpha$ quantifies subdiffusion processes. Here, we propose $\gamma$ and $\alpha$ maps obtained in a controlled heterogeneous phantom, comprised of packed micro-beads in water and in excised human meningiomas. In few words, $\alpha$ maps represent the multi-scale spatial distribution of the disorder degree in the system, while $\gamma$ maps are influenced by local internal gradients, thus highlighting the interface between compartments characterized by different magnetic susceptibility. $\gamma$ maps were already obtained by means of $A D$ stretched exponential imaging and $\alpha$-type maps have been recently achieved for fixed rat brain with the aim of highlighting the fractal dimension of specific brain regions. However, to our knowledge, the maps representative of the spatial distribution of $\alpha$ and $\gamma$ obtained on the same controlled sample and in the same excised tissue have never been compared. Moreover, we show here, for the first time, that $\alpha$ maps are representative of the spatial distribution of the disorder degree of the system.

In a first phase, $\gamma$ and $\alpha$ maps of controlled phantom characterized by an ordered and a disordered rearrangement of packed micro-beads of different sizes in water and by different magnetic susceptibility $(\Delta \chi)$ between beads and water were obtained. In a second phase, we investigated excised human meningiomas of different consistency.

Results reported here, obtained at 9.4T, show that $\alpha$ and $\gamma$ maps are characterized by a different image contrast. Indeed, unlike $\gamma$ maps, $\alpha$ maps are insensible to $(\Delta \chi)$ and they are sensible to the disorder degree of the microstructural rearrangement. These observations strongly suggest that $\mathrm{AD}$ indices $\alpha$ and $\gamma$ reflect some additional microstructural information which cannot be obtained using conventional diffusion methods based on Gaussian diffusion. Moreover, $\alpha$ and $\gamma$ maps obtained in excised meningiomas seem to provide more microstructural details above those obtained with conventional DTI analysis, which could be used to improve the classification of meningiomas based on their consistency.
\end{abstract}

(c) 2012 Elsevier Inc. All rights reserved.

\section{Introduction}

During the past few decades, diffusion-weighted imaging (DWI) [1] and diffusion tensor imaging (DTI) [2] have become popular methods to provide quantitative information on microscopic characteristics and abnormalities of human tissues $[3,4]$. Despite that, DWI and DTI have intrinsic methodological limitations which impoverish

* Corresponding author. CNR-IPCF Physics Department Sapienza University of Rome, P.le Aldo Moro 5, 00185 Rome Italy. Tel.: + 390649913928 ; fax: + 390649913928 E-mail address: silvia.capuani@roma1.infn.it (S. Capuani). the sensitivity and specificity of these diagnostic tools. One of these limitations is due to the assumption that diffusion in biological tissues is Gaussian, namely that exists a linear relation between the mean square displacement (MSD) of diffusing molecules and time (t) during which diffusion occurs. Indeed, dynamics of water in living systems can be hindered and/or restricted by confining boundaries in multiple compartments leading to a deviation from the linear relation between MSD of diffusing particles and $t[5,6]$. Moreover, some biological tissues, such as the cerebral ones, can be described in terms of a multi-scale and multi-level complex system, in which a complex hierarchical topology resembling fractal folding can be found $[7,8]$. 
In such kind of media, water molecules can be entrapped in microstructures and obstructions. Moreover, irregular and chaotic travel paths of water molecular displacement can be observed. In this case, the MSD as a function of $t$, is characterized by a power law: $\operatorname{MSD}(\mathrm{t}) \propto \mathrm{t}^{\mathrm{v}}$ (where $\mathrm{v}$ is the dynamic exponent of the transport process, or scaling index) which characterizes anomalous diffusion (AD) processes. The case $\mathrm{v}>1$ describes superdiffusive, while $\mathrm{v}<1$ describes subdiffusive processes $[9,10]$. In this scenario, AD or nonGaussian diffusion methods may, in principle, provide more or different microstructural information which couldn't be obtained using conventional diffusion methods based on Gaussian diffusion.

In human tissue DWI, the signal as a function of the b-value, has been shown to deviate from monoexponential decay, in particular, at high values of the diffusion-weighting factor, b. This phenomenon, observed in brain more than ten years ago [11,12] is termed nonGaussian diffusion.

Recently, we introduced a novel method [13] to obtain microstructural information from heterogeneous media by means of diffusion methods based on non-Gaussian diffusion theory. The approach utilizes the theory of the Continuous Time Random Walk (CTRW) [10] as an effective approach to describe the features of non normal, or anomalous diffusion $[9,10]$.

According to the CTRW model, the motion propagator of $\mathrm{AD}$ is defined as the solution of fractional diffusion equations. These equations introduce two parameters, the fractional exponent in time $\alpha$ and the fractional exponent in space $\mu=2 \gamma$ which are the time and space derivatives fractional orders, respectively. We measured both the spatial AD index $\gamma$ and the temporal AD index $\alpha$, by using spectroscopic pulse gradient stimulated echo (PGSTE) methods [13] showing that $\gamma$ quantifies pseudo-superdiffusion processes $[13,14]$ while $\alpha$ quantifies subdiffusion processes [13]. Moreover, $\gamma$ depends on local magnetic susceptibility $(\Delta \chi)[13,14]$ or on effective internal gradients [15-17] and it is equivalent to the stretching parameter of the $\mathrm{AD}$ stretched exponential imaging method, recently developed to investigate brain tissue [18-20]. Conversely, $\alpha$ provides information on the disorder degree of the system and does not depend on $\Delta \chi$. To the best of our knowledge, $\alpha$ and $\gamma$ maps obtained on the same controlled sample, have never been compared.

Our aim was to assess the potential of spatio-temporal anomalous diffusion imaging in reflecting additional microstructural information that cannot be obtained using conventional diffusion methods based on Gaussian diffusion.

To achieve this goal, $\gamma$ and $\alpha$ weighted images obtained in a controlled heterogeneous phantom and in excised human meningiomas at high magnetic field (9.4T) were analyzed and compared between themselves and with conventional mean diffusivity (MD) maps. Our results show that $\alpha$ maps represent, in an efficient way, the spatial distribution of the disorder degree in the system, while $\gamma$ maps confirm their property of reflecting local $\Delta \chi$, thus highlighting the interface between compartments characterized by different $\Delta \chi$. Data reported here strongly suggest that $\alpha$ and $\gamma$ imaging could be useful tools to obtain additional information compared to those obtainable from conventional DTI methods, for characterizing tissue microstructures. Here, we tested in vitro the potential of spatio-temporal anomalous diffusion in discriminating between hard and soft excised human meningiomas [21].

\subsection{Theory}

One of the most interesting features of pulse field gradient (PFG) sequences used to perform diffusion studies, is that the measured signal is proportional to the Fourier transform (FT) of the motion propagator (MP), where, the MP is proportional to the probability of finding a diffusing particle in a position $\mathrm{r}$ at a time $\mathrm{t}$.

By taking into account the theory of CTRW [10,13], MP is defined as the solution of fractional diffusion equations. These equations introduce two parameters, the fractional exponent in time $\alpha$ and the fractional exponents in space $\gamma$, which are the time and space derivatives fractional orders. Briefly, to investigate sub-diffusive processes for which the mean-square displacement (MSD) of diffusing particles grows sub-linearly in time, it is possible to assume the following asymptotic behavior for the FT of MP:

$W(k, t) \propto \exp \left(-K_{\alpha} k^{2} t^{\alpha}\right)$ when $k^{2} \ll 1 /\left(K_{\alpha} t^{\alpha}\right)$

where $k=1 /(2 \pi) g \delta \Upsilon$ with: $g$, the diffusion gradient strength, $\delta$, the gradient pulse duration, $\Upsilon$ the nuclear gyromagnetic ratio and $0<\alpha<1$. Conversely, to study super-diffusive processes characterized by a divergence of the jump length variance, it is possible to use the following function:

$W(k, t) \propto \exp \left(-K^{2 \gamma} k^{2 \gamma} t\right)$

where $0<\gamma<1$.

As a consequence, the specific AD behaviour of water in each investigated sample, is described by the two $\alpha$ and $\gamma$ indices that, in principle, reflect a different information about the microstructural rearrangement of investigated samples. From Eq. (1) it is obvious that in a PGSTE sequence, by changing the diffusion time $\Delta$ at a fixed value of $g$ the signal decay can be fitted by Eq. (1), for evaluating $\alpha$. On the other hand, changing the $g$ value at fixed $\Delta$, by fitting the signal decay as a function of k to Eq. (2), yields $\gamma$.

In the effective approach proposed here, the underlying premise is that MSD may grow sublinearly with time for a limited interval of time. Indeed, it is well known that diffusion is anomalous for all times if particles diffuse in a suitable hierarchy of binding sites and are not in thermal equilibrium with traps [22].

In many natural systems, a range of heterogeneity length scales $\leq \lambda$ (where $\lambda$ is the largest heterogeneity length scale) exists, which can produce a broad distribution of diffusing particles stopping times. Therefore, the transport on a scale $L \ll \lambda$ and $L \gg \lambda$ evolves to normal behavior because the medium appears homogeneous and the transport process is well described by the linear growth of MSD with time. Conversely, on a scale $L \leq \lambda$ the transport reflects the medium heterogeneities and evolves to a more complex behavior, known as anomalous diffusion.

As well demonstrated by many studies based on Monte Carlo simulations of particle diffusion in 2D [22-28] and 3D crowded media [29], particle diffusion in media comprised of mono-sized obstacles uniformly and randomly distributed, is characterized by the following representative behaviors:

1. for very short times diffusion is normal, i.e. the measured macroscopic diffusion coefficient is constant and equal to that of particle diffusion in an homogeneous medium without any crowding effects, $\mathrm{D}_{0}$;

2. for short times diffusion is anomalous, i.e. the measured macroscopic diffusion coefficient is time-dependent and is well described by the relation: $D(t) \propto^{\alpha-1}$, with $0<\alpha<1$;

3. for long times diffusion is normal again, with a constant macroscopic diffusion coefficient, $\mathrm{D}_{\infty}<\mathrm{D}_{0}$.

Transition from anomalous to the normal diffusion regime is characterized by the crossover time $t^{*}$, corresponding to the largest heterogeneity length scale, $\lambda: \lambda \sim\left(t^{*} D_{\infty}\right)^{1 / 2}$.

Therefore, the premise at the basis of the approach adopted in this work is that, in the investigated systems, anomalous diffusion is expected to occur for diffusion times $\mathrm{t} \leq \mathrm{t}^{*}$.

Moreover, the crossover from anomalous to normal diffusion, which is expected for diffusion times $t>t^{*}$ cannot be observed in the NMR experiments performed in this study due to intrinsic limitations of the experimental technique. In particular, in the systems 
investigated, the longitudinal relaxation time, $T_{1}$, of water diffusing molecules throw polystyrene micro-beads, or in excised human meningiomas, is smaller than the cutoff time scale $t^{*}$. This means that for the samples investigated in this study by NMR diffusion measurements, the long-time behavior known as the tortuosity limit cannot be observed.

\section{Material and methods}

\subsection{Controlled phantom}

Styrene bead suspensions (Microbeads AS, Norway) in de-ionized water and Tween 20 surfactant at high sphere packing approximately equal to $70 \%$ and $\mathrm{TiO}_{2}$ beads in water were used to investigate $\mathrm{AD}$ in heterogeneous systems. Beads characterized by mean diameters of $0.05,5,6$, and $10 \mu \mathrm{m}$, were used to produce phantoms in which water can probe microstructures dimension typically observed in biological tissues. Moreover $\Delta \chi$ between polystyrene beads and water is approximately $\left|\Delta \chi_{m}\right|=\left|\chi_{m}^{\mathrm{H} 2 \mathrm{O}}-\chi_{m}^{\text {Polystyrene }}\right|=1.59 \times 10^{-6}$ in SI units, while $\Delta \chi$ between $\mathrm{TiO}_{2}$ and water is approximately $10^{-5}$ (SI). Specifically, glass microcapillaries with $0.7 \mathrm{~mm}$ inner diameter were filled with: free water (capillary number 1 , in reference image of Fig. 1); capillary number 2 is filled with a mixture of $6 \mu \mathrm{m}$ and $10 \mu \mathrm{m}$ beads suspended in water. As a consequence it represents a more disordered system [13]; capillary number 3 is filled with $\mathrm{TiO}_{2} 5 \mu \mathrm{m}$ beads monodispersed in water; capillary number 4 is filled with $\mathrm{TiO}_{2}$ $0.05 \mu \mathrm{m}$ beads monodispersed in water; capillaries 5 and 6 are filled with $6 \mu \mathrm{m}$ polystyrene beads monodispersed in water. These six microcapillaries were immersed in a $8 \mathrm{~mm}$ NMR tube (Fig. 1) containing $10 \mu \mathrm{m}$ packed polystyrene beads in water (ROI 7 in reference image). In summary, capillaries 3, 4,5 and 6, and ROI 7, are representative of ordered systems. Capillaries 3 and 4 are characterized by strong internal gradients. Capillary 2 is the only one representative of a disordered system, and capillary 1 is representative of a system characterized by normal or Gaussian diffusion.

\subsection{Excised brain tissues}

One patient, bearing a brain meningioma presents in different areas, underwent an MR DTI investigation at $3 \mathrm{~T}$ (Philips Achieva) prior to surgery, including diffusion-weighted sequences (single-shot spinecho echoplanar (SSh-SE-EPI) with TE/TR $=109 / 5000 \mathrm{~ms}$, slice thickness $4 \mathrm{~mm}$, in plane resolution $2.2 \mathrm{~mm}^{2}$ ) acquired in 15 non collinear directions at 2 different b-values $\left(1000,2500 \mathrm{~s} / \mathrm{mm}^{2}\right)$ plus one image at $\mathrm{b}=0$. Twenty-four contiguous axial slices were collected with $\mathrm{NS}=$

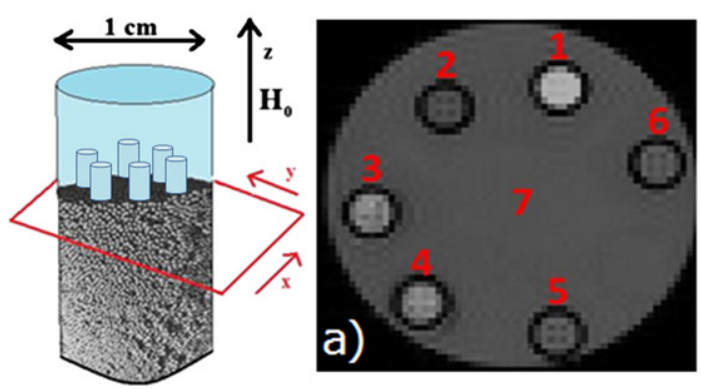

Fig. 1. Details of controlled phantom used to mimic media with low and high level of disorder and with different $\Delta \chi_{m}$. An axial low resolution T2-weighted image is displayed in a) to describe phantom details. Capillary number 1 is filled with free water and Tween 20 surfactant; capillary number 2 is filled with a mixture of 6 and $10 \mu \mathrm{m}$ beads of polystyrene polydispersed in water to simulate a disordered system; capillary number 3 is filled with $\mathrm{TiO}_{2} 5 \mu \mathrm{m}$ beads monodispersed in water; capillary number 4 is filled with $\mathrm{TiO}_{2} 0.05 \mu \mathrm{m}$ beads monodispersed in water; capillaries 5 and 6 are filled with $6 \mu \mathrm{m}$ polystyrene beads monodispersed in water; ROI 7 in reference T2-w image is characterized by $10 \mu \mathrm{m}$ polystyrene beads monodispersed in water. Please note that, in this phantom, capillary 2 only is representative of a disordered system.
2. An 8-channel SENSE head coil was used. After the surgery, excised specimens of meningioma, named $\mathrm{C}$ and $\mathrm{E}$ (Fig. 2, upper panel) were selected on the basis of their consistency. For microscopic diagnosis, representative tumour samples from the extracted samples $\mathrm{C}$ and $\mathrm{E}$ were fixed in $10 \%$ buffered formalin and embedded in paraffin; five micrometer-thick serial sections were stained with Haematoxylin \& Eosin or employed for immunohistochemistry. Histologically representative images of the main characteristic features of sample $\mathrm{C}$ and $\mathrm{E}$ are displayed in Fig. 2 (bottom panel).

Sample $C$ was characterized by a major zone of soft tissue with a minor zone with fibres. It is representative of a meningioma with a reduced consistency, characterized by the presence of sheets and lobules of the typical meningothelial cells in a vague papillary arrangement. Conversely, sample E was characterized by a harder consistency than sample $C$. Indeed, it is characterized by extensive areas of hypocellular fibrous hyalinization starting from the perivascular space, with rare residual small groups of meningothelial cells. From the point of view of neurosurgery, soft meningiomas are those characterized by reduced consistency, which can be aspired as opposed to hard meningiomas that cannot be aspired [21]. To investigate excised meningiomas, we placed samples $\mathrm{C}$ and $\mathrm{E}$, along with a solution of $4 \%$ paraformaldehyde and phosphate-buffered saline (PBS) solution for fixation, to preserve microstructure rearrangements [30] in two $8 \mathrm{~mm}$ NMR tube. Samples $\mathrm{C}$ and E were also investigated by optical microscopy and sectioned, after the in vitro NMR examinations.

\subsection{MRI}

All measurements were performed on a Bruker 9.4T Avance system, operating with a micro-imaging probe $(10 \mathrm{~mm}$ internal diameter bore) and equipped with a gradient unit characterized by a maximum gradient strength of $1200 \mathrm{mT} / \mathrm{m}$, and a rise time of $100 \mu \mathrm{s}$.

An imaging version of Pulse Gradient Stimulated Echo (PGSTE) sequence with $\Delta / \delta=40 / 4.4 \mathrm{~ms}$, diffusion gradients along $\mathrm{x}, \mathrm{y}$ and $\mathrm{z}$ axis, $\mathrm{TR}=5 \mathrm{~s}$, slice thickness $1 \mathrm{~mm}, \mathrm{FOV}=8 \mathrm{~mm}$ and 10 values of $\mathrm{g}$ from 28 to $224 \mathrm{mT} / \mathrm{m}$, i.e. effective b values range from 500 to $6500 \mathrm{~s} /$ $\mathrm{mm}^{2}$, plus one effective $\mathrm{b}=230 \mathrm{~s} / \mathrm{mm}^{2}$ for selecting $\mathrm{b}=0$ image (where effective $\mathrm{b}$ is due to both diffusion and imaging gradients, while $\mathrm{b}$ is due to only diffusion gradient), was used to obtain MD at different b-values and $\gamma$ maps (by fitting Eq. (2) to the data). Moreover $\Delta$ variable PGSTE imaging sequence with $\delta=4.4 \mathrm{~ms}, \mathrm{~g}=$ $40 \mathrm{mT} / \mathrm{m}, \mathrm{TR}=5 \mathrm{~s}-\Delta$, slice thickness $1 \mathrm{~mm}, \mathrm{FOV}=8 \mathrm{~mm}$ and 10 values of $\Delta$ in the range $20-420 \mathrm{~ms}$ (i.e. effective b values range from 500 to $6500 \mathrm{~s} / \mathrm{mm}^{2}$ ), was used for collecting data to fit to Eq. (1) for extracting $\alpha$ map. Differently from $\gamma$ map, to obtain an $\alpha$ map without any other influence from relaxation effects, it is necessary to divide each signal $\mathrm{S}(\mathrm{g}, \Delta)$ obtained using $\mathrm{g}=40 \mathrm{mT} / \mathrm{m}$ and a certain value of $\Delta$, to the signal $\mathrm{S}(\Delta)$ obtained using the corresponding $\Delta$.

The mean values of $\alpha(\mathrm{M} \alpha)$ and $\mu(\mathrm{M} \gamma)$ were obtained by averaging: $M \alpha=1 / 3 \Sigma_{i=x, y, z}(\alpha)_{i}$ and $M \mu=1 / 3 \Sigma_{i=x, y, z}\left(\gamma_{e f f}\right)_{i}$. Because phantoms used in our experiments are characterized by a cylindrical geometry, we measured $\mathrm{MD}, \mathrm{M} \gamma$ and $\mathrm{M} \mu$ along $\mathrm{x}, \mathrm{y}$ and $\mathrm{z}$ axis only. However, in more complex geometries, a tensor analysis should be used [19].

All fitting procedures were performed by means of LevenbergMarquardt algorithm using homemade scripts in MATLAB 2009b.

\section{Results and discussion}

$\mathrm{M} \gamma, \mathrm{M} \alpha$ and the conventional MD map obtained from the controlled phantom illustrated in Fig. 1, are displayed in Fig. 3 (A, B and $C$, respectively). Moreover in Table $1, M \gamma$ and $M \alpha$ mean values and their standard deviation (SD), obtained in each ROI marked in Fig. 1a) from three consecutive slices, are listed. The $M \gamma$ and $M \alpha$ maps are "grainy" compared to a conventional MD image, due to the short 

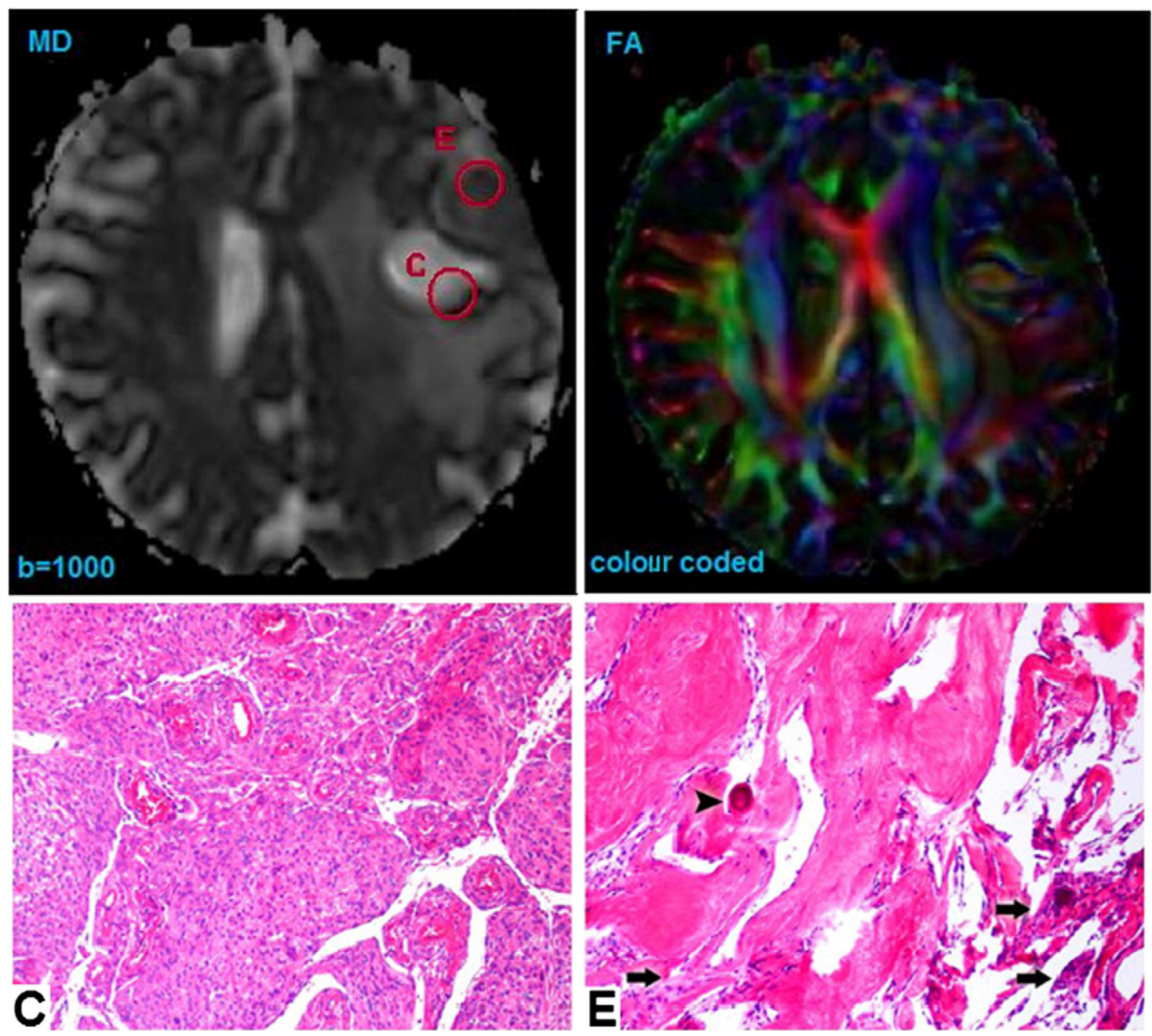

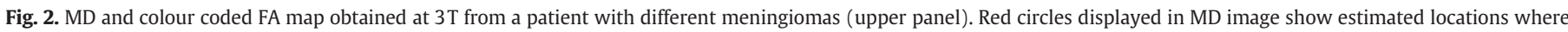

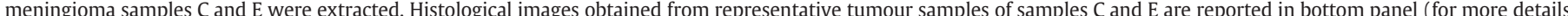

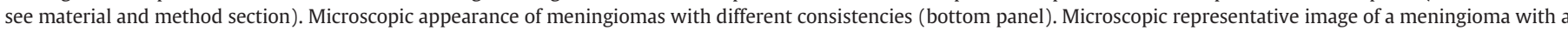

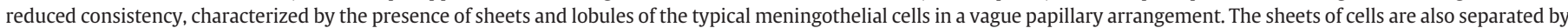

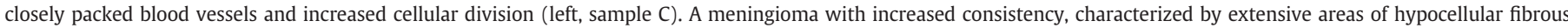

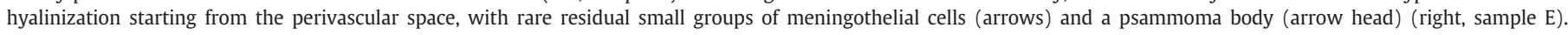
Haematoxylin \& Eosin stain, original magnification, $\times 100$.

range of the possible $\gamma$ and $\alpha$ values. Indeed, in the type of phantoms used in our experiments, which are comprised of packed beads and water, $\gamma$ values run from 0.7 to 1 (as you can see in Fig. 3A), while $\alpha$ values run from 0.85 to 1 (Fig. $3 \mathrm{~B}$ ). Conversely MD values run from $0.1 \times 10^{-4} \mathrm{~mm}^{2} / \mathrm{s}$ to $8 \times 10^{-4} \mathrm{~mm}^{2} / \mathrm{s}$. Apart the rendering of the gray scale, MD map, which is based on estimation of Gaussian diffusion, shows a different image contrast when compared to both, $\mathrm{M} \gamma$ and $\mathrm{M} \alpha$ maps. Specifically, M $\gamma, \mathrm{M} \alpha$ and MD measurements are not correlated to each other. Capillary 1, shows the highest M $\gamma$ and $M \alpha$ values, close to 1, according to Gaussian diffusion behavior of free water. Conversely, capillaries 3 and 4 show the lowest M $\gamma$ values. Finally capillary 2, which is the only capillary filled with beads of two
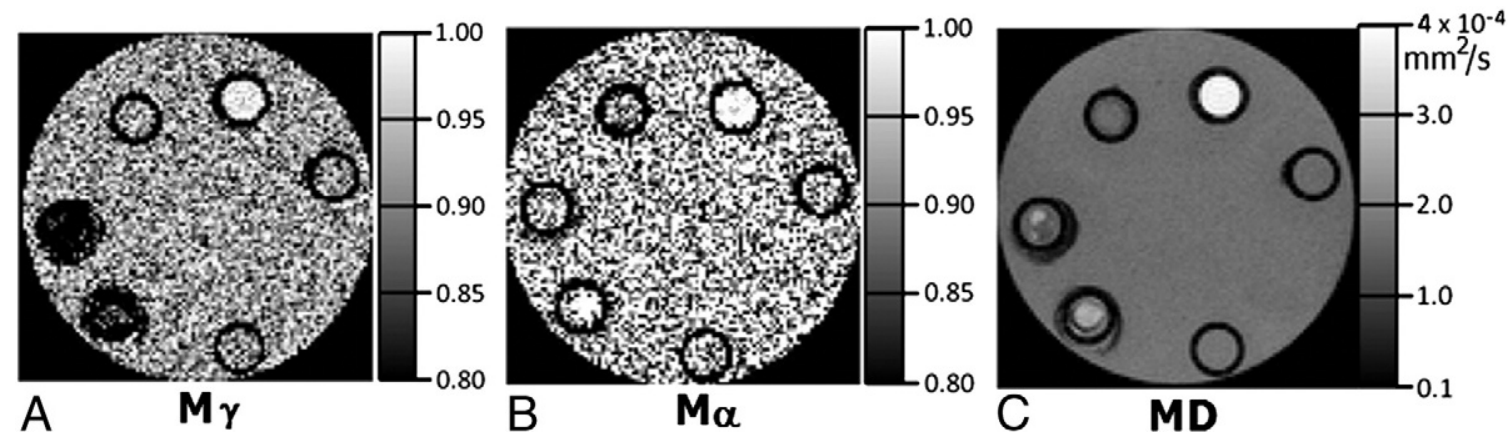

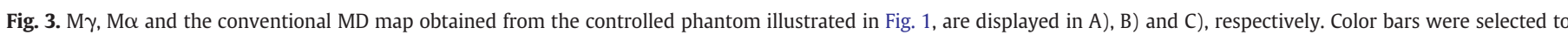
emphasize gray level differences between capillaries different from that filled with free water solution (capillary 1 in Fig. 1 ). 
Table 1

$\mathrm{MD}, \mathrm{M} \gamma, \mathrm{M} \alpha$ mean $\pm \mathrm{SD}$ values obtained from three consecutive slices of each map reported in Fig. 3 in ROIs depicted in the T2-weighted image displayed in Fig. 1.

\begin{tabular}{llll}
\hline ROI & MD $\left(10^{-4} \mathrm{~mm}^{2} / \mathrm{s}\right)$ & M & M \\
\hline 1 & $7.95 \pm 0.70$ & $0.97 \pm 0.03$ & $0.98 \pm 0.02$ \\
2 & $2.54 \pm 0.38$ & $0.88 \pm 0.02$ & $0.85 \pm 0.01$ \\
3 & $2.69 \pm 0.44$ & $0.67 \pm 0.03$ & $0.89 \pm 0.01$ \\
4 & $3.58 \pm 0.54$ & $0.69 \pm 0.05$ & $0.92 \pm 0.03$ \\
5 & $2.93 \pm 0.42$ & $0.90 \pm 0.02$ & $0.90 \pm 0.01$ \\
6 & $2.69 \pm 0.44$ & $0.90 \pm 0.02$ & $0.90 \pm 0.01$ \\
7 & $3.03 \pm 0.21$ & $0.91 \pm 0.02$ & $0.91 \pm 0.02$ \\
\hline
\end{tabular}

different sizes, shows the lowest $M \alpha$ value, according to the theory of disordered systems [13].

Maps in Fig. 3A and 3B, are likely to detect changes in local $\Delta \chi$ and in disorder degree, respectively. In other words, $\mathrm{M} \gamma$ is representative of the spatial distribution of the local $\Delta \chi$, while $M \alpha$ is representative of the spatial distribution of the disorder degree of the system.
By observing M $\gamma$ map and its values listed in the Table 1, it is possible to check the ability of $\mathrm{M} \gamma$ map in detecting differences in samples containing different local $\Delta \chi$. In the $M \gamma$ map there is a better discrimination of capillaries filled with mono-dispersed $\mathrm{TiO}_{2}$ beads in water than those filled with polystyrene micro-beads in water. This is due to the high sensitivity of $M \gamma$ to $\Delta \chi$ which is about one order of magnitude higher in $\mathrm{TiO}_{2}$ beads in water sample than that in polystyrene beads in water sample. According to our previous results $[13,14]$, data reported in Fig. 3A) confirms that M $\gamma$ contrast depends on both the water dynamics and the $\Delta \chi$.

With regard to M $\alpha$ contrast, $\alpha$ value does not depend on $\Delta \chi$, but it depends on the spatial distribution of the disorder degree of media. Indeed $\alpha$-image contrast is approximately equal to 0.9 in all sample locations filled up with high packed monodispersed beads, which are representative of ordered systems [13]. Conversely, $\alpha$ value evaluated in the capillary number 2 , in which mixed bead sizes were used to mimic an ad hoc region of disorder, shows the lowest value in the $\mathrm{M} \alpha$ map. As a consequence, $\mathrm{M} \alpha$ map displayed in Fig. 3B, strongly
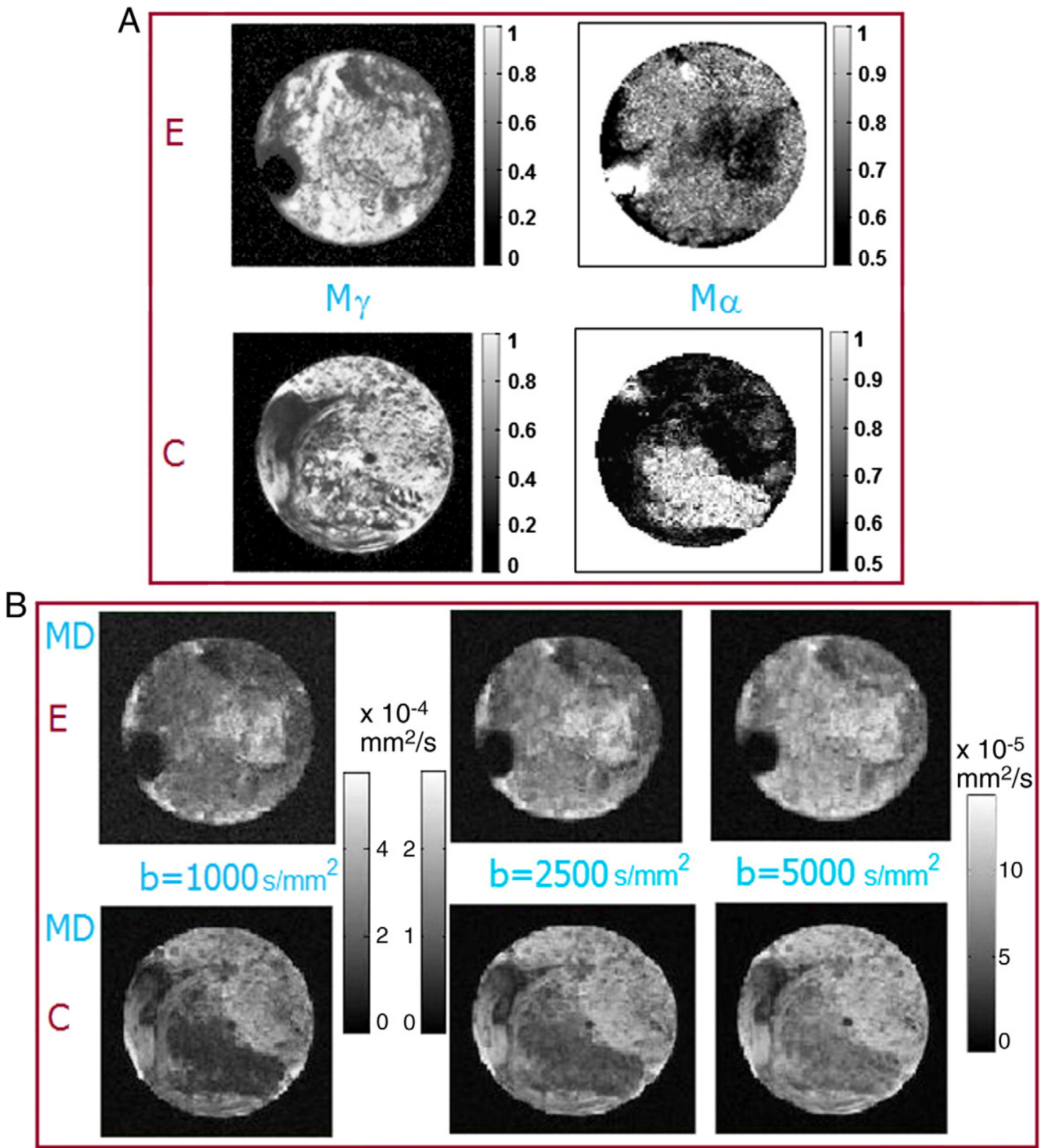

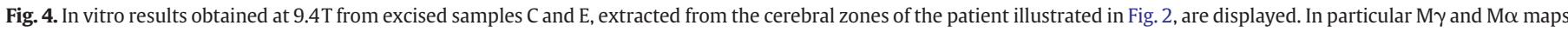

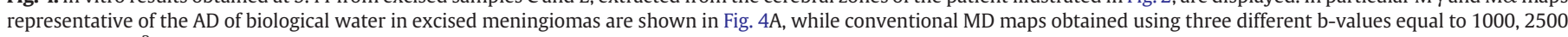
and $5000 \mathrm{~s} / \mathrm{mm}^{2}$ are displayed in Fig. 4B. Color bars were selected to emphasize gray level differences among tissue zones in each sample. 
suggests that $\mathrm{AD}$ index $\alpha$ provides a new type of image contrast, different from that provided by conventional diffusion procedures based on Gaussian diffusion (i.e. MD maps) and different from that provided by $\gamma$ stretched exponential model.

$\mathrm{M} \gamma$ and $\mathrm{M} \alpha$ maps representative of the $\mathrm{AD}$ of biological water in excised brain tissue samples $\mathrm{C}$ and $\mathrm{E}$ are shown in Fig. 4A, together with conventional MD images obtained using three different $\mathrm{b}$-values (Fig. 4B). Comparing results in Fig. 4A with histological images displayed in Fig. 2, we can confirm the observations made to the results displayed in Fig. 3. Indeed, M $\gamma$ map of sample $\mathrm{C}$, which is characterized by the presence of a lot of meningothelial cells, highlights the boundaries and interfaces between cells/extracellular space, tissue/vessels and low/high tissue cellularity; in other words $\mathrm{M} \gamma$ map of sample C offers a better detection than that achievable by MD images of the boundaries and interfaces between substances characterized by a different $\Delta \chi$, such as tissue/vessels and low/high tissue cellularity. Conversely, $\mathrm{M} \alpha$ map of sample $\mathrm{C}$ seems to be less affected by local $\Delta \chi$ due to blood and/or tissue cellularity Unlike M $\gamma$ image, $M \alpha$ map discriminates between two zones in sample C, characterized by a more disordered ( $\alpha$ less than 0.65 ) and a less disordered (or more ordered) tissue ( $\alpha$ higher than 0.8 ). These two zones seem to reflect approximately the two areas in MD images characterized by a less and a more slow dynamics (Fig. 4B, bottom panel). A deeper investigation of samples $C$, carried out after the NMR experiments, showed that the area of sample $C$ that was characterized by the highest $\alpha$ value and the lowest MD value, was occupied by a high anisotropic fibrous tissue, i.e., by a tissue characterized by an ordered structure.

Moreover sample E, which is characterized by a more ordered and homogeneous fibrous structure than sample $\mathrm{C}$, shows a higher mean $\alpha$ values when compared to sample $\mathrm{C}$.

$\mathrm{M} \alpha$ results, are in agreement with the results obtained by Ozarslan et al. [8]. These authors have recently reported the first map representative of the spatial distribution of the fractal dimension in fixed rat brain. In particular, the parameter that quantifies the fractal dimension, is proportional to the $\alpha$ parameter [8,13]. Ozarslan et al. [8] use acquisitions at varying $\Delta$ (as in our approach) but they used also acquisition at different q values to obtain fractal dimension maps. In particular, the fractal dimension, $\mathrm{d}_{\mathrm{f}}$, which describes the scaling of the mass of the environment with distance, is the combination of two exponents characterizing the diffusion process: the fractal dimension of the diffusion process, $d_{w}$ and the spectral or fractal dimension, $d_{s}$, which quantify the scaling behavior of the density of states for the Laplacian operator. Generally speaking, $d_{w}$ defines the scaling behavior of the MSD with time and it is strictly related to the $\alpha$ exponent of the present work: $d_{w}=2 \alpha$, while $d_{s}$ defines the scaling behavior of the return to the origin probability (RTOP) with time: $R T O P \propto t^{-\mathrm{d}_{s} / 2}$. In deterministic fractals, the two dynamics exponents are related to $d_{\mathrm{f}}$ through the simple relationship: $d_{f}=\frac{d_{w} d_{s}}{2}$.

As a consequence, the difference between $M \alpha$ and fractal dimension maps lies in the fact that the maps reported by Ozarslan et al. [8] are aimed to map the fractal dimension in cerebral tissues, while the $\mathrm{M} \alpha$ maps reported here are representative of the spatial distribution of the degree of disorder.

Finally, by observing maps displayed in Fig. 4, unlike MD maps, even at high b values, $M \alpha$ and M $\gamma$ maps, seem to be able to highlight some additional microstructural details to finely discriminate the two samples $\mathrm{E}$ and $\mathrm{C}$ characterized by different consistency.

\section{Conclusion}

In recent years, several approaches have been proposed to quantify non-Gaussian [31-35] and $\mathrm{AD}$ processes in heterogeneous systems $[8,13,14,18-20,36-41]$. In this paper, for the first time, we show and compare anomalous subdiffusive $\mathrm{M} \alpha$ maps together with anomalous pseudo-superdiffusion $\mathrm{M} \gamma$ and conventional MD maps obtained from the same samples (controlled phantom and excised brain tissues). In particular, here we presented the imaging version of our recent spectroscopic results [13] which indicated $\alpha$, as an AD parameter to quantify the disorder degree of the system. Moreover, they indicated $\gamma$, as an $\mathrm{AD}$ parameter to quantify restricted multicompartimentalized diffusion processes in which local $\Delta \chi$ at the interface between compartments is present $[13,14]$. Indeed real superdiffusion processes are considered impossible for water molecules in the type of systems used in this work. However, due to abrupt changes of magnetic susceptibility at interfaces, their images can mimic a superdiffusion process in NMR measurements.

$\mathrm{M} \gamma$ and $\mathrm{M} \alpha$ maps illustrated here, which are representative of the spatial distribution mapping of the local heterogeneities and of the degree of the structural disorder, respectively, strongly suggest to provide some additional information about the microstructural rearrangements of heterogeneous media in which water diffuses, compared to conventional MD maps. We have tested the potential of $\mathrm{M} \alpha$ and $\mathrm{M} \gamma$ as new complementary tool to potentiate the sensitivity and specificity of diagnosis based on diffusion protocols, in excised meningiomas of different consistency. Our results and observations suggest that this AD approach might be potentially useful for in vivo application, to detect different and more microscopic features of pathological lesions compared to those observable using Gaussian DTI approach.

\section{References}

[1] Le Bihan D. Molecular diffusion nuclear magnetic resonance imaging. Magn Reson Quarterly 1991;7:1-30.

[2] Basser PJ. Inferring microstructural features and the physiological state of tissues from diffusion-weighted images. NMR Biomed 1995;8:333-44.

[3] Bozzali M, Cherubini A. Diffusion tensor MRI to investigate dementias: a brief review. Magn Reson Imaging 2007;25:969-77.

[4] Koh D-M, Collins DJ. Diffusion-weighted MRI in the body: applications and challenges in oncology. Am J Roentgenol 2007;188:1622-35.

[5] Assaf Y, Freidlin RZ, Rohde GK, Basser PJ. New modeling and experimental framework to characterize hindered and restricted water diffusion in brain white matter. Magn Reson Med 2004;52:965-78.

[6] Grebenkov DS. NMR survey of reflected Brownian motion. Rev Mod Phys 2007;79: 1077-137.

[7] Santamaria F, Wils S, De Schutter E, Augustine GJ. The diffusional properties of dendrites depend on the density of dendritic spines. Eur J Neurosci 2011:1-8.

[8] Özarslan E, Shepherd TM, Koay CG, Blackband SJ, Basser PJ. Neuroimage 2012;60: 1380-93.

[9] Ardelean I, Kimmich R. Principles and unconventional aspects of NMR diffusometry. Ann Rep NMR Spectr 2003;49:43-115.

[10] Metzler R, Klafter J. The random walk's guide to anomalous diffusion: a fractional dynamics approach. Phys Rep 2000;339:1-77.

[11] King MD, Houseman J, Gadian DG, Connelly A. Localized q-space imaging of the mouse brain. Magn Reson Med 1997;38:930-7.

[12] Assaf Y, Cohen Y. Non mono-exponential attenuation of water and N-acetyl aspartate signals due to diffusion in brain tissue. J Magn Reson 1998;131:69-85.

[13] Palombo M, Gabrielli A, De Santis S, Cametti C, Ruocco G, Capuani S. Spatiotemporal anomalous diffusion in heterogeneous media by NMR. J Chem Phys 2011;135:034504.

[14] Palombo M, Gabrielli A, De Santis S, Capuani S. The $\gamma$ parameter of the stretchedexponential model is influenced by internal gradients: validation in phantoms. J Magn Reson 2012;216:28-36.

[15] De Santis S, Rebuzzi M, Di Pietro G, Maraviglia B, Capuani S. In vitro and in vivo MR evaluation of internal gradient to assess trabecular bone density. Phys Med Biol 2010;55:5767-85.

[16] Rossi C, Boss A, Martirosian P, Steidle G, Capuani S, Claussen CD, et al. Influence of steady background gradients on the accuracy of molecular diffusion anisotropy measurements. Magn Reson Imaging 2008;26:1250-8.

[17] Fasano F, Capuani S, Hagberg GE, Branca RT, Indovina I, Castriota-Scanderbeg A et al. Intermolecular double quantum coherences (iDQc) and diffusion-weighted imaging (DWI) imaging of the human brain at $1.5 \mathrm{~T}$. Magn Reson Imaging 2003;21:1241-9.

[18] Bennett KM, Schmainda KM, Bennett RT, Rowe DB, Lu H, Hyde JS. Characterization of continuously distributed cortical water diffusion rates with a stretchedexponential model. Magn Reson Med 2003;50:727-34.

[19] De Santis S, Gabrielli A, Bozzali M, Maraviglia B, Macaluso E, Capuani S. Anisotropic anomalous diffusion assessed in the human brain by scalar invariant indices. Magn Reson Med 2011;65:1043-52.

[20] Hall MG, Barrick TR. Two-step anomalous diffusion tensor imaging. NMR Biomed 2012;25:286-94

[21] Yamaguchi N, Kawase T, Sagoh M, Ohira T, Shiga H, Toya S. Prediction of consistency of meningiomas with preoperative magnetic resonance imaging. Surg Neurol 1997;48:579-83. 
[22] Saxton MJ. A biological interpretation of transient anomalous subdiffusion. II. reaction kinetics. Biophys J 2008:94:760-71.

[23] Saxton MJ. Lateral diffusion in an archipelago. The effect of mobile obstacles. Biophys J 1987;52:989-97.

[24] Saxton MJ. Lateral diffusion in a mixture of mobile and immobile particles. A Monte Carlo study. Biophys J 1990;58:1303-6.

[25] Saxton MJ. Lateral diffusion in an archipelago. Dependence on tracer size. Biophys J 1993;64:1053-62.

[26] Saxton MJ. Anomalous diffusion due to obstacles. A Monte Carlo study. Biophys J 1994;66:394-401.

[27] Saxton MJ. Anomalous diffusion due to binding: a Monte Carlo study. Biophys J 1996:70:1250-62.

[28] Saxton MJ. A biological interpretation of transient anomalous subdiffusion. I. Qualitative model. Biophys J 2007;92:1178-91.

[29] Vilaseca E, Isvoran A, Madurga S, Pastor I, Garces JL, Mas F. New insights into diffusion in 3D crowded media by Monte Carlo simulations: effects of size, mobility and spatial distribution of obstacles. Phys Chem Chem Phys 2011;13: 7396-407.

[30] Kim TH, Zollinger L, Shi XF, Rose J, Jeong E-K. Diffusion tensor imaging of ex vivo cervical spinal cord specimens: the immediate and long-term effects of fixation on diffusivity. Anat Rec (Hoboken) 2009;292(2):234-41.

[31] Özarslan E, Shemesh N, Basser PJ. A general framework to quantify the effect of restricted diffusion on the NMR signal with applications to double pulsed field gradient NMR experiments. J Chem Phys 2009;130:104702.

[32] Shemesh N, Özarslan E, Basser PJ, Cohen Y. Measuring small compartmental dimensions with low-q angular double-PGSE NMR: the effect of experimental parameters on signal decay. J Magn Reson 2009;198:15-23.
[33] Shemesh N, Özarslan E, Adiri T, Basser PJ, Cohen Y. Noninvasive bipolar doublepulsed-field-gradient NMR reveals signatures for pore size and shape in polydisperse, randomly oriented, inhomogeneous porous media. J Chem Phys 2010;133:044705.

[34] Jensen JH, Helpern JA, Ramani A, Lu H, Kaczynski K. Diffusional kurtosis imaging: the quantification of non-gaussian water diffusion by means of magnetic resonance imaging. Magn Reson Med 2005;53:1432-40.

[35] Novikov DS, Kiselev VG. Effective medium theory of a diffusion-weighted signal. NMR Biomed 2010:23:682-97.

[36] Özarslan E, Basser PJ, Shepherd TM, Thelwall PE, Vemuri BC, Blackband SJ. Observation of anomalous diffusion in excised tissue by characterizing the diffusion-time dependence of the MR signal. J Magn Reson 2006;183: $315-23$.

[37] Magin RL, Abdullah O, Baleanu D, Zhou XJ. Anomalous diffusion expressed through fractional order differential operators in the Bloch-Torrey equation. J Magn Reson 2008;190:255-70.

[38] De Santis S, Gabrielli A, Palombo M, Maraviglia B, Capuani S. Non-Gaussian diffusion imaging: a brief practical review. Magn Reson Imaging 2011;29(10): $1410-6$.

[39] Bennett KM, Hyde JS, Rand SD, Bennett R, Krouwer HGJ, Rebro KJ, et al. Intravoxel distribution of DWI decay rates reveals C6 glioma invasion in rat brain. Magn Reson Med 2004;52:994-1004.

[40] Zhou XJ, Gao O, Abdullah O, Magin RL. Studies of anomalous diffusion in the human brain using fractional order calculus. Magn Reson Med 2010;63:562-9.

[41] Kwee TC, Galbán CJ, Tsien C, Junck L, Sundgren PC, Ivancevic MK, et al. Intravoxel water diffusion heterogeneity imaging of human high-grade gliomas. NMR Biomed 2010;23:179-87. 